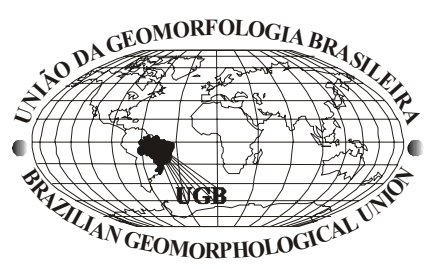

\title{
GEOMORFOLOGIA COSTERAY EXPLOTACIÓN DE ARENADE PLAYA EN LA PROVINCIA DE BUENOS AIRES Y SUS CONSECUENCIAS AMBIENTALES
}

Silvia C. Marcomini

Departamento de Geología, Facultad de Ciencias Exactas y Naturales, Universidad de Buenos Aires, Cp 1428. Buenos Aires, Argentina.

scm@gl.fcen.uba.ar

Rubén López.

Departamento de Geología, Facultad de Ciencias Exactas y Naturales, Universidad de Buenos Aires, Cp 1428. Buenos Aires, Argentina

\section{Resumen}

El presente trabajo tiene como objetivo principal caracterizar el medio físico de las áreas costeras, con el fin determinar los impactos en la hidrodinámica litoral, a mediano y corto plazo, generado por la explotación de arena de playa en las principales localidades costeras de la provincia de Buenos Aires, entre cabo San Antonio y Necochea. Para tal fin se describen las explotaciones mineras de mayor magnitud llevadas a cabo a lo largo de la costa de la provincia de Buenos Aires y se analiza su efecto para cada morfología costera. En todos los casos las explotaciones han ocasionado un deficit de arena en la corriente de deriva litoral, lo que hace que la erosión se traslade como una onda en el sentido de la deriva litoral, es decir hacia el norte para estas costas. El tipo de costa que recibe el impacto de esta onda condiciona el grado de erosión a la que este sujeta. En costas acantiladas cohesivas las explotaciones han producido un importante incremento en las tasas de retroceso de los acantilados y pérdida de playa. En costas definidas como de tipo B (con desarrollo de bermas) los efectos erosivos de la explotación, sumados a otros factores antrópicos, han afectado fundamentalmente las morfologías de playa, mientras que en las de tipo A (sin desarrollo de bermas), el efecto no solo ha alcanzado la playa sino que también ha provocado retroceso de la línea de costa. Asimismo se determinó que las tasas de retroceso de la costa se aceleran o retardan en igual medida durante la explotación y recuperación respectivamente; es decir que la recuperación por interrupción de las actividades mineras es semejante en magnitud a la erosión inducida durante la explotación.

Palabras clave: Minería, arena de playa, impacto ambiental

\begin{abstract}
The sand mining in coastal areas has been a problem in continuos conflict. The growth of cities requires increasing quantities of sand for building, that is usually extracted from beach, with intensification of erosion problems. The main objective of this paper is geomorphological and environmental characterization of the northeastern Buenos Aires coast, between San Antonio cape and Necochea, to identify the impacts generated by the exploitation of sand from the beach on the coastal communities. Major beach sand mining activities carried out along the coast are described, as well as their effects on each coastal type. In all cases, beach sand mining caused a deficit of sediments for the longitudinal currents, with exacerbation of erosional effects to downdrift. The morphology of the coast plus the impact of essentially erosional waves defined the beach erosion pattern, wich influences coastal community. In cohesive cliffy coasts beach mining increased the coastal recession rates and loss of sand cover over the wave-cut platforms. In type B coasts (with development of berms), the sand mining induced changes on the beach morphology and decrease on the beach level. In type A coasts (without berms), the erosional effect of the sand mining not only altered the beach declivity and morphology, but also induced a backward movement of the dune toe.
\end{abstract}

Keywords: Mining, beach sand, environmental impact. 


\section{Introduccion}

El hombre ha producido cambios importantes en el litoral Atlántico de la provincia de Buenos Aires durante los últimos 40 años, como consecuencia de un rápido incremento poblacional acompañado de una deficiente planificación y falta de políticas de manejo adecuadas para las áreas costeras. La falta de sustentabilidad entre las actividades humanas y las características geomorfológicas del sistema costero han intensificado la erosión en los centros urbanos de mayor desarrollo.

La sumatoria de impactos ambientales ha provocado en el medio físico importantes cambios en las variables que regulan el equilibrio del ecosistema costero. Es así que los fenómenos de erosión de playas, retroceso de costas, alteración del paisaje y deterioro de los acuíferos, se reconocen en gran parte de las costas de la provincia de Buenos Aires. Esta situación condiciona seriamente el desarrollo económico-social de estas localidades, cuya principal actividad es el turismo, basado en el recurso natural que ofrecen sus playas.

La explotación de arena en áreas costeras ha sido siempre un problema de conflicto continuo. El crecimiento de la urbanización incrementó el uso de áridos para la construcción, que fueron explotados de arena de playa y duna, intensificando los problemas de erosión en los municipios costeros. En la actualidad, resulta imperioso contar con sitios de disponibilidad de arena no solo para la construcción, sino que también para efectuar episodios y proyectos de remediación de las playas afectadas por estos efectos erosivos. En otros países del mundo la principal fuente de abastecimiento para repoblación de playas es la explotación de arena en la playa sumergida, a profundidades superiores a lo $20 \mathrm{~m}$. En Argentina este tipo de explotación resulta muy costosa, ya que requiere la movilización de dragas y de estudios de bancos arenosos submarinos como posibles fuentes de explotación.

El conocimiento de la disponibilidad de arena como recurso en áreas costeras es fundamental para el desarrollo de cualquier comunidad. Proyectos semejantes han sido llevados a cabo por organismos estatales en otros lugares del mundo, no solo para abastecer la industria de la construcción, sino que también para conocer los recursos disponibles para remediar las playas, y mitigar fenómenos erosivos. Como ejemplo puede mencionarse el desarrollado en los Estados Unidos para la costa del Golfo de México por el U.S. Geological Survey (Geologic assesments and characterization of marine sand resources - Gulf of Mexico Region) por Williams y Cichon (1993), cuyo objetivo principal fue localizar y evaluar los recursos de arena marinos e interpretar su origen.

Los trabajos que describen el efecto de la explotación de áridos en la hidrodinámica litoral son escasos. En la bibliografía internacional el efecto de la extracción de arena sobre la playa anterior y sumergida fue estudiado por Hilton $(1989,1990)$ y Hesp y Hilton (1996) para las costas autralianas, Tsurusaki et al. (1988), por Uda et al. (1986) para las costas del Japón y por Anctil y Ouellet (1990) para Canada.

En la Argentina Isla y Fasano (1987) alertaron sobre los impactos generados por las explotaciones de arena sobre la playa y propusieron distintas recomendaciones mineras para minimizar los efectos adversos de la explotación. Marcomini y López (1999) investigaron el efecto producido por la extracción de arena de playa de una cantera ubicada en el Partido de Lobería sobre la dinámica, morfología y textura de las playas. Asimismo, evaluaron la recuperación de las playas en la zona de extracción y en sectores aledaños, luego del cese de las tareas de explotación en junio de 1997.

El objetivo del presente trabajo consiste en evaluar el impacto que han tenido las actividades mineras en los distintos tipos de costa que se ubican a lo largo del litoral de la provincia de Buenos Aires entre Cabo San Antonio y Necochea y estimar la capacidad soporte del sistema. Otro de los puntos a abordar es el análisis de las características texturales de la arena de playa, y sus condiciones para ser empleadas en hormigón por su compatibilidad con las normas IRAM (Instituto Argentino de Racionalización de Materiales).

\section{Explotacion, Sitios y Historia}

El importante crecimiento edilicio de las comunidades costeras desde la década del 70 aumentó bruscamente la demanda de arenas para la construcción, y por consiguiente se produjo una sobrexplotación del recurso disponible de menor costo, la arena de playa. Las explotaciones no solo abastecieron los Municipios Costeros, sino que también localidades cercanas tales como General Lavalle, Dolores, Maipú, Ayacucho, Balcarce, Tres Arroyos y Bahía Blanca. En 1986 se crea la Dirección de Geología, Minas y Aguas Subterraneas de la provincia de Buenos Aires (DIGMAS), organismo que propone llevar un control de las explotaciones litorales en el dominio Público (por debajo del nivel de las pleamares). Este organismo no tiene injerencia sobre las explotaciones en la duna costera, ya que pertenece al dominio privado. Con el objeto de evitar la erosión el organismo permitía a cada permisionario extraer un volumen de hasta $100 \mathrm{~m}^{3} /$ día de un espesor máximo de $0,5 \mathrm{~m}$, sobre una parcela de 50 a $100 \mathrm{~m}$. A pesar de ello la explotación indiscriminada se intensificó hasta fines de la década del 80 , cuando el efecto de tormentas extraordinarias pusieron en evidencia el déficit de saturación de arena en la deriva litoral, generando una intensa erosión en los frentes costeros de numerosas localidades que se declararon en estado de alerta y comenzaron a tomar conciencia de la gravedad del problema.

La explotación de arena de playa finalmente fue prohibida en los partidos de Mar Chiquita, Pueyrredón, 
General Alvarado y Lobería (decreto ley 8756) y en los partidos de La Costa, Pinamar y Villa Gesell (Disposición 2/ 95). No obstante ello, existen explotaciones clandestinas en las distintas localidades, tales como Punta Médanos, San Clemente, Mar de Ajó y Nueva Atlántis.

En la actualidad Caballé et al. (2005) estimaron que la producción actual de arena no supera las 200.000 t/año.

La prohibición de la extracción de arena sin considerar la posibilidad de explotación de otros sitios alternativos ha generado problemas sociales entre los que se destacan la falta de reinserción laboral de los pobladores que efectúan explotaciones clandestinas y el aumento del costo de la construcción por la falta de arena con características apropiadas.

En la figura 1 se muestran los sitios correpondientes a antiguas explotaciones de arena del noreste de la provincia de Buenos Aires

1) Municipio de la Costa: En esta localidad la explotación ha sido intensiva a lo largo de toda la costa, en especial en Mar de Ajó (El Silvio), Las Toninnas y San Clemente del Tuyú (Filsa). En la construcción de la ruta interbalnearia, entre
General Lavalle y Punta Médanos se emplearon 1.000.000 de $\mathrm{m}^{3}$ de arena obtenidos principalmente de las playas y dunas costeras de las localidades de San Clemente del Tuyú, Las Toninas y Mar de Ajó entre 1972 y 1976 (López y Marcomini, 2003). En 1993 la Provincia prohibió la explotación de arena de playa.

2) Villa Gesell: al sur de la localidad existía una explotación intensiva. Las concesiones otorgadas en Villa Gesell declararon volúmenes de arena con magnitudes de extracción próximas a la deriva neta, del orden de $\operatorname{los} 400.000 \mathrm{~m}^{3}$ (Isla y Fasano, 1987).

3) Mar Chiquita: La explotación fue prohibida a mediados de 1980, luego de que la localidad homónima registrara intensos problemas de erosión.

4) Partido de General Pueyrredón: En épocas prósperas de la construcción se estimó que el volumen extraído en el partido habría alcanzado unos $600.000 \mathrm{~m}^{3}$ anuales lo que equivaldría a la deriva neta (Schnack, et al 1983). En 1985 1986 se prohibió la extracción de arena de playa en este distrito y para compensar este hecho en 1988 se autorizó la

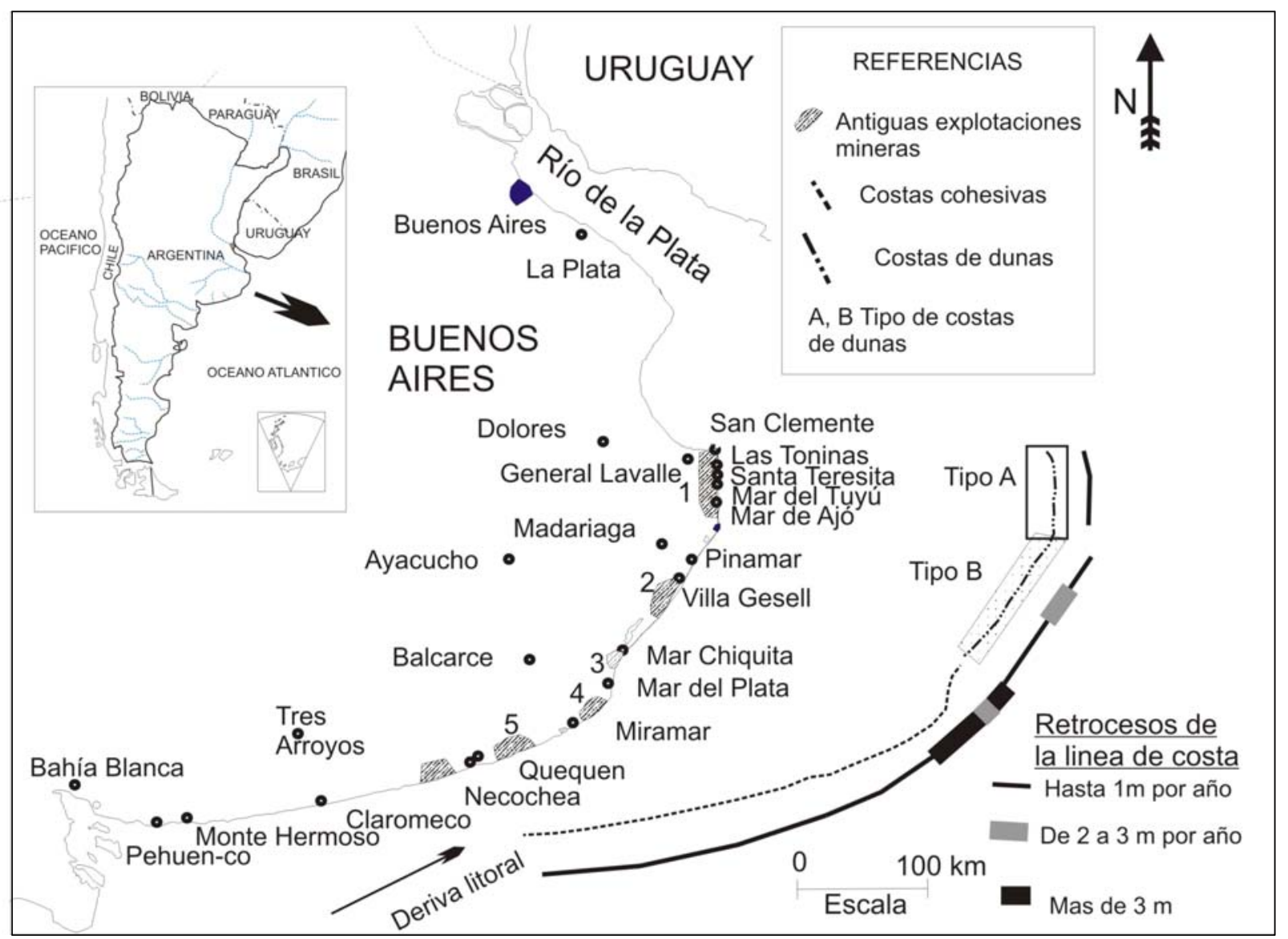

Figura 1 - Mapa de ubicación donde se muestran los sitios correspondientes a antiguas explotaciones mineras. Pueden observarse los distintos tipos de costa que caracterizan la región, los rangos de retroceso y la deriva litoral neta. 
explotación en el Partido de Lobería. El sector de mayor explotación en General Pueyrredón correspondía al Faro San Jacinto.

5) Partido de Lobería: Las mayores explotaciones se realizaron en Centinela del Mar, en una cantera ubicada al pie de una costa acantilada. La actividad minera afectó una extensión de $1.800 \mathrm{~m}$ de playa, sobre una superficie de 200.000 $\mathrm{m}^{2} \mathrm{y}$ un volumen estimado en $150.000 \mathrm{~m}^{3}$ de arena. Las tareas de extracción minera se realizaban principalmente de los sectores de berma y playa frontal y abarcaban una extensión de $900 \mathrm{~m}$ a lo largo de la línea de ribera. La actividad minera cesó en junio de 1997, luego de inducir serios problemas deerosión a las localidades ubicadas deriva abajo (Centinela del Mar, Mar del Sur y Miramar).

6) Partido de Necochea: Existió una explotación importante de arena de playa al sur de Necochea en el balneario Los Angeles entre 1997 y 1998 se explotaron aproximadamente $32.000 \mathrm{~m}^{3}$.

Si bien las explotaciones de minería de playa han sido prohibidas en toda la costa, aún pueden observarse extracciones de importante magnitud, tal como se distingue en la figura 2, donde se observan camiones extrayendo y acopiando arena de playa en el sector de Punta Médanos (Municipio de la Costa), en marzo de 2006.

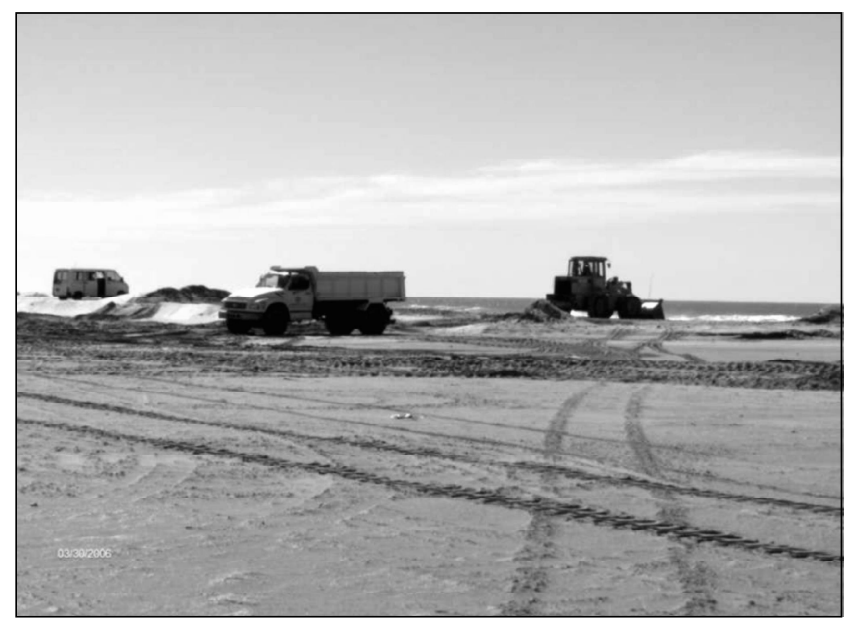

Figura 2 - Explotación de arena de playa en camiones en Punta Médanos en marzo de 2006.

También, a pesar de la prohibición siguen existiendo explotaciones menores, de particulares, con carros (Figura 3).

\section{Metodologia}

Se efectuó el análisis morfológico de las siguientes localidades: San Clemente del Tuyu, Las Toninas, Mar del Tuyú, Mar de Ajó, Nueva Atlántis, Punta Médanos, Pinamar, Villa Gesell, Mar Chiquita, Mar del Sur y Quequén, ubicadas

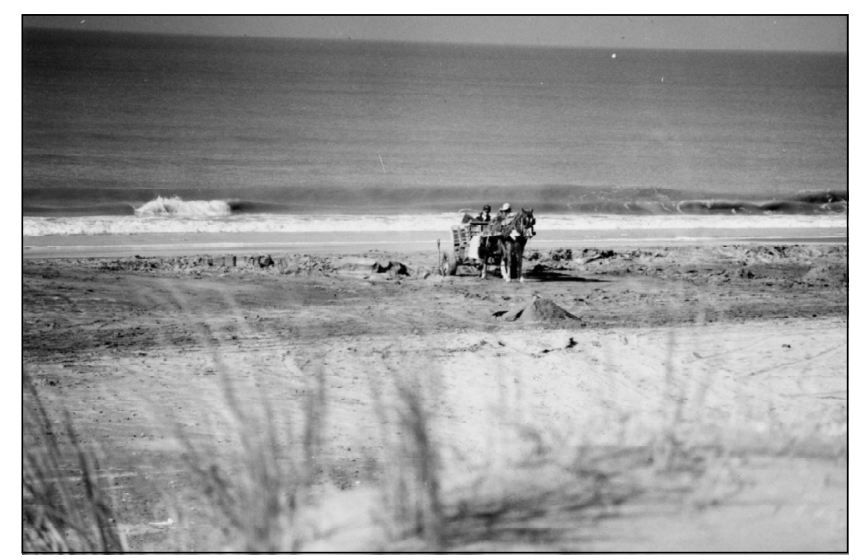

Figura 3 - Extracción de arena en carros particulares, Municipio de la Costa.

de norte a sur respectivamente.

Se emplearon fotos aéreas a escala 1:20.000 pertenecientes a GEODESIA y al Servicio de Hidrografia Naval.

Se efectuaron y compararon perfiles de playa para evaluar el estado de alteración de las playas y las condiciones hidrodinámicas y se extrayeron muestras superficiales de arena de la playa anterior, sector intermareal.

Se monitorearon los sectores correspondientes a las antiguas explotaciones mineras para determinar la situación actual de las localidades aledañas.

\section{Metodologia}

Se efectuó el análisis morfológico de las siguientes localidades: San Clemente del Tuyu, Las Toninas, Mar del Tuyú, Mar de Ajó, Nueva Atlántis, Punta Médanos, Pinamar, Villa Gesell, Mar Chiquita, Mar del Sur y Quequén, ubicadas de norte a sur respectivamente.

Se emplearon fotos aéreas a escala 1:20.000 pertenecientes a GEODESIA y al Servicio de Hidrografia Naval.

Se efectuaron y compararon perfiles de playa para evaluar el estado de alteración de las playas y las condiciones hidrodinámicas y se extrayeron muestras superficiales de arena de la playa anterior, sector intermareal.

Se monitorearon los sectores correspondientes a las antiguas explotaciones mineras para determinar la situación actual de las localidades aledañas.

\section{Resultados}

\section{Dinámica costera}

Las mareas son micromareales con un régimen de tipo semidiurno con desigualdades diurnas cuya amplitud media es de $0,82 \mathrm{~m}$. Las playas están expuestas a la acción directa 
del oleaje con dos direcciones principales de incidencia de los trenes de ola, las provenientes principalmente del sur y sudeste, que origina una corriente de deriva litoral neta hacia el norte (Figura 1). La hidrodinámica litoral está regulada por las fases paroxísmicas asociadas a condiciones de tormentas extraordinarias que involucran un incremento del nivel del mar, las cuales no poseen una periodicidad probable aunque tienen mayor recurrencia durante el verano. En la costa de Buenos Aires estos registros corresponden a sudestadas o tormentas del oeste que provocan ascensos de hasta $1,5 \mathrm{~m}$ sobre el nivel de marea esperado.

\section{Geomorfología}

La provincia de Buenos Aires posee dos tipos de costas: costas de dunas y costas cohesivas.

Las primeras se extienden al norte de la laguna de Mar Chiquita (Figura 1). Se caracterizan por un campo de dunas de $3,5 \mathrm{~km}$ de ancho. La costa presenta una duna costera activa que regula el equilibrio de la playa durante las tormentas. Por sus rasgos morfológicos, las costas de dunas estudiadas, pueden dividirse en dos sectores, A y B (Figura 1).

En el sector A la duna costera no posee gran desarrollo y alcanza alturas de hasta $2 \mathrm{~m}$. (Figura 4 A y D). Las playas se caracterizar por un perfil de configuración lineal con un ancho de playa que varía de 40 a $70 \mathrm{~m}$. La pendiente media es de 0,028 $\left(1^{\circ} 36^{\prime}\right)$. No se observa la presencia de bermas estables ni estacionales. Las mareas de sizigias llegan hasta el pie de la duna, generando escarpas de erosión luego de las sudestadas. Frecuentemente se observa la presencia de barras de lavado en la playa frontal, con anchos de 15 a $20 \mathrm{~m}$ y altura de $25 \mathrm{~cm}$.

En el sector B (Figura 4 B y E), las playas poseen pendientes de 1 a $2^{\circ} 30^{\prime}$ y están constituidas por arena mediana a fina (Figura 4). El perfil de playa natural tiene: 1) una berma estable de aproximadamente $40 \mathrm{~m}$ de longitud y $0,75 \mathrm{~m}$ de altura localizada en el sector de playa distal; 2) una o dos bermas transicionales que se adosan a la anterior de 20 a 25 $\mathrm{m}$ de longitud y $0,5 \mathrm{~m}$ de altura. Estas últimas son sobrepasadas por las olas durante mareas extraordinarias o en condiciones de tormenta (storm surges), donde pasarían a ser afectadas por la acción de lavado. El ancho de playa varía de 80 a $150 \mathrm{~m}$ y la pendiente media es de $0,014\left(0^{\circ} 48^{\prime}\right)$. La duna costera posee alturas de aproximadamente $5 \mathrm{~m}$ desde el pie de duna y terminan directamente en la playa distal. Por lo general en el sector costero la duna no tiene una morfología muy definida.

Las costas cohesivas (Figura 4 C y F), son de erosión y muestran en su mayor extensión acantilados activos labrados sobre arenas no consolidadas (dunas) o sobre limolitas calcáreas con intercalaciones de calcretes "tosca". Un campo de dunas colgadas, por lo general inactivo se desarrolla en el sector superior de los acantilados. Las playas asociadas a acantilados activos son angostas y desarrollan configuraciones variadas. Generalmente carecen de bermas estables y están asociadas a plataformas de abrasión. Las playas de mayor desarrollo por lo general presentan pendientes variables entre 0,73 y 1,59 grados y anchos que oscilan entre $97 \mathrm{~m}$ y $270 \mathrm{~m}$. Los perfiles de playa se caracterizan por presentar una berma estable de 50 a $60 \mathrm{~m}$ de longitud y de aproximadamente $0,6 \mathrm{~m}$ de altura localizada a una cota de $3 \mathrm{~m}$ sobre el nivel medio de bajamares. Frecuentemente se reconocen barras de lavado de 20 a $30 \mathrm{~m}$ de longitud y de 0,2 a $0,3 \mathrm{~m}$ de altura. El ancho de la zona de rompiente (breaker zone), oscila de 20 a $50 \mathrm{~m}$ y la zona de desplazamiento (surf), abarca una extensión de aproximadamente $100 \mathrm{~m}$. La amplitud de ambas zonas se encuentran controladas por la existencia de plataformas rocosas de extensión variada.

\section{Erosión costera}

La erosión producida por el hombre es directa cuando existe alguna degradación sobre la morfología natural e indirecta cuando una acción producida por el mismo altera algún parámetro que, si bien no se percibe en el momento, producirá una modificación a futuro. Entre los factores de erosión directa la explotación de arena ha sido una de las principales causas. Al anterior se le han sumado la depredación y degradación de dunas costeras, el trazado de avenidas, las modificaciones en el escurrimiento superficial, la evacuación de excedentes pluviales al mar y la circulación de vehículos en la zona de playa y duna.

Las causas de erosión antrópica indirecta son: interrupción de la dinámica litoral, por construcción de puertos, escolleras, espigones, y muelles, forestación y urbanización de campos de dunas interiores, implantación de especies foráneas en el cordón de dunas costeras, generación de barreras eólicas, edificación en sectores cercanos a la playa, loteos incompatibles con la morfología cuya calidad sostiene el recurso económico más importante que es el turismo.

Estos factores han traído como consecuencia la aceleración localizada en la pérdida crónica de territorio a mediano plazo, evidenciada por retroceso en la línea de costa y la disminución de la superficie de la playa.

Se han identificado distintas velocidades de retroceso de la costa en de la provincia de Buenos Aires (Figura 1), las tasas mayores rondaron los $5 \mathrm{~m} / \mathrm{año}$ en Mar Chiquita (Isla 1997) y al sur de Mar del Plata en la zona Faro- Playa San Jacinto entre 1970 y 1982 (Schnack et al. 1983). En este último sector debido fundamentalmente a las explotaciones mineras en la zona de playa que luego se prohibieron en el año 1986 a partir de cuando, comienzan a disminuir las tasas de retroceso a 3,33 m/año(1985-1988) y 2,50 m/año(1988-1992), (Cionchi et al. 1993), (Figura 5B). 

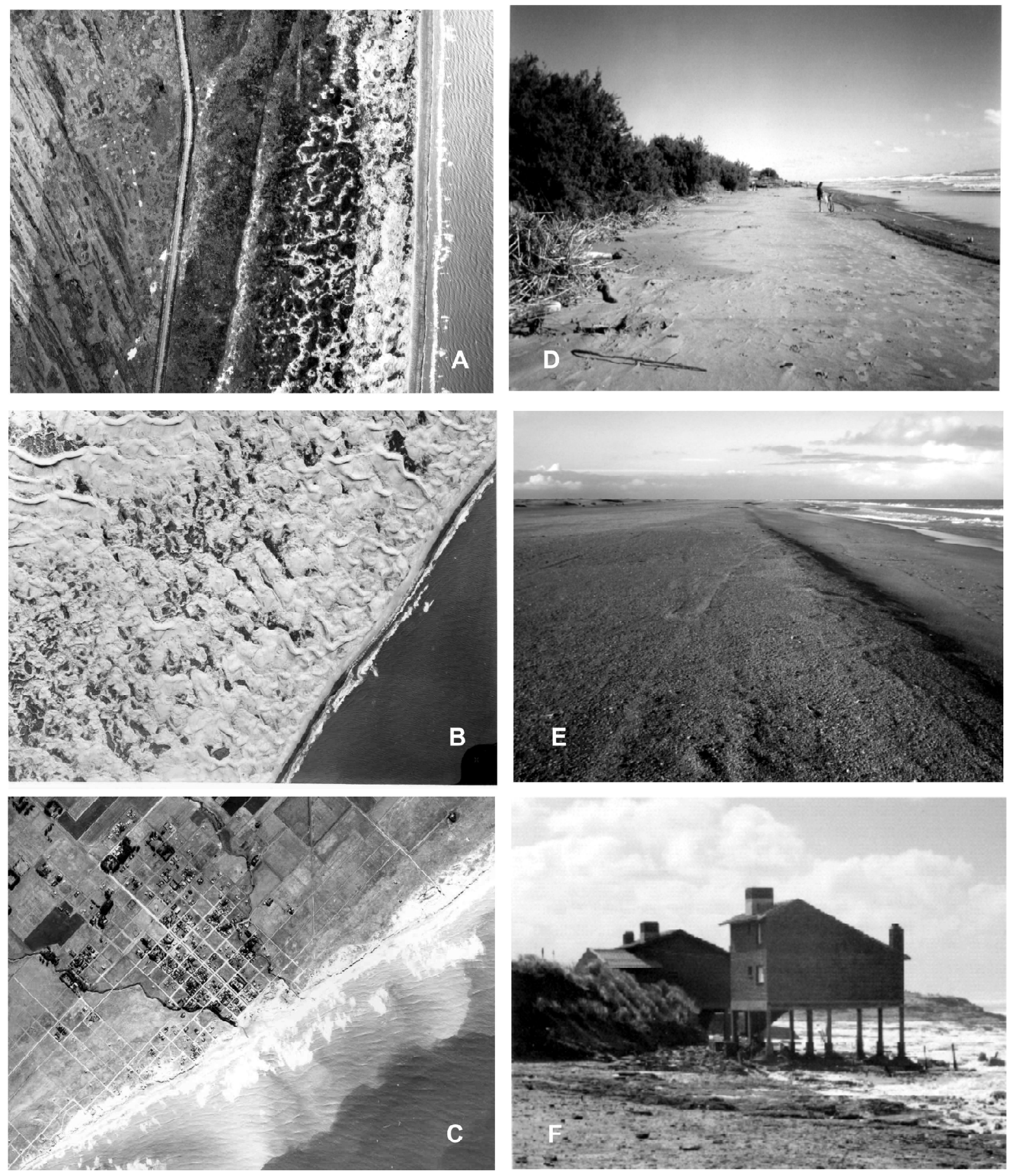

Figura 4 - Lámina que muestra las características morfológicas de la costa y playa.

Fotos aéreas de los distintos tipos de costa: A. Municipio de la Costa (Tipo A), B. Punta Médanos (Tipo B) y C. Mar del Sur (costa cohesiva). D. Vista de la playa al sur de San Clemente. E. Situación natural de playa al sur de Punta Médanos, F. Vista de la playa en costas cohesivas (Mar del Sur).

Otras localidades ubicadas en costas de dunas han evidenciado erosión antrópica mediante aceleraciones críticas en los retrocesos asociados a centros urbanos como es el caso de Villa Gesell (Figura 5A), Pinamar, Mar del Tuyú y Las Toninas, alcanzando retrocesos de $2,45 \mathrm{~m} / \mathrm{año}$ (Marcomini y López 1997) en la primera localidad. 

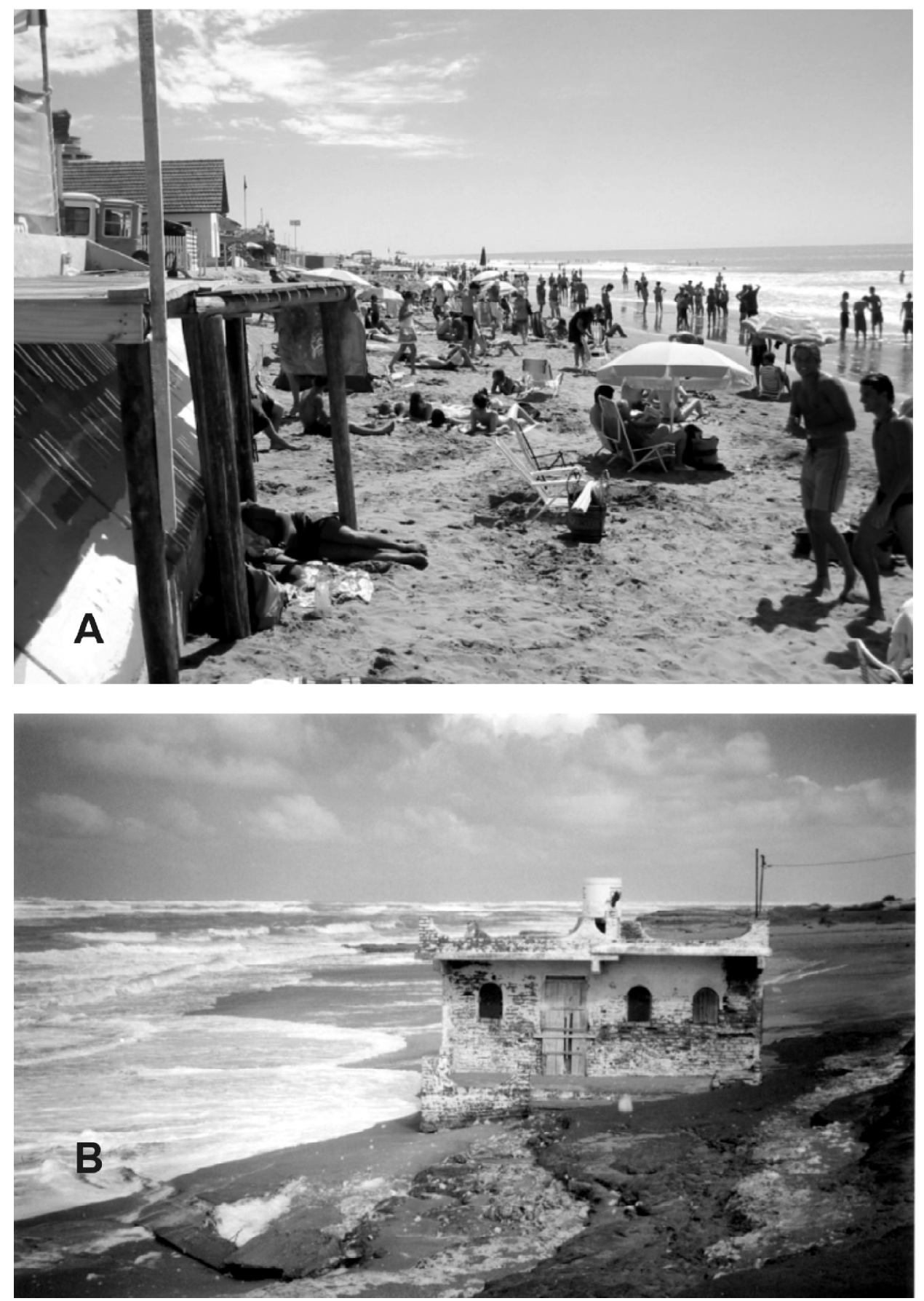

Figura 5 - Localidades afectadas por erosión antrópica inducida en ambos tipos de costas. A. Villa Gesell (costa de dunas tipo B), luego de la tormenta del 28 de Diciembre de 2003. B. Mar del Sur (costa cohesiva).

\section{Sedimentología y aptitud de los depósitos de playa para la construcción}

Las playas del área en estudio poseen distinto tamaño de grano dependiendo del tipo de costa. Las playas correspondientes a las costas de dunas de tipo A presentan por lo general distibuciones unimodales, con modas predominantes en el intevalo de clase de 3 phi. Se componen de arena fina, asimetría negativa, y la selección es buena $(0,35-0,50$ phi).

Las playas que componen las costas de dunas tipo B se caracterizan por tener poblaciones polimodales con una 
moda principal más recurrente en el intervalo de 3-3,5 phi y una moda secundaria entre 0 y 1,5 phi. Están compuestas por arena fina a mediana, con asimetría generalmente negativa y selección moderada (0,71-1,00 phi).

Las playas que constituyen las costas cohesivas poseen por lo general tamaño de grano más grueso y las variaciones texturales son muy marcadas a lo largo del tiempo, en especial luego de fuertes tormentas. Los sedimentos están constituidos por poblaciones polimodales con moda principal entre 2 y 2,5 phi y moda secundaria en 0,5 phi. La media corresponde a arena mediana (1-2 phi), son pobremente seleccionados y la asimetría es negativa.

La normativa que rige la granulometría de los áridos para la construcción está expresada en la norma IRAM 1627. La misma, diferencia entre sedimentos para ser utilizados en la construcción como agregados finos, gruesos o totales.

La normativa indica que el agregado fino podría obtenerse por mezcla de 2 o más arenas de distinta granulometría.
En la figura 6 se pueden observar los límites establecidos para las categorías aptas (A y B) y en obras de "tipo corriente", donde el control de calidad de hormigones se realice en obra (norma IRAM 1627 art. 3.2.5). No obstante ello se podrían aceptar ocasionalmente los sedimentos cuyas granulometrías no superen el límite estipulado por la curva C.

De la figura se puede observar que ninguna de las arenas de playa cumple con la norma IRAM 1627.

Las curvas que más se aproximan son las correspondientes a los sedimentos de playa de Villa Gesell, cuyos valores quedan comprendidos entre las curvas $\mathrm{A}$ y $\mathrm{C}$, con un $28 \%$ entre $\mathrm{A}$ y B, un $50 \%$ entre $\mathrm{B}$ y $\mathrm{C}$, y un $12 \%$ nuevamente entre Ay B para los sedimentos más finos.

Los sedimentos correspondientes las playas de Mar del Sur (costa cohesiva) serían aptos bajo ciertas restricciones, ya que su intervalo de clase cae entre las curvas B y C, o sea que podrían ser empleadas solo para hormigones en obras pequeñas y con control.

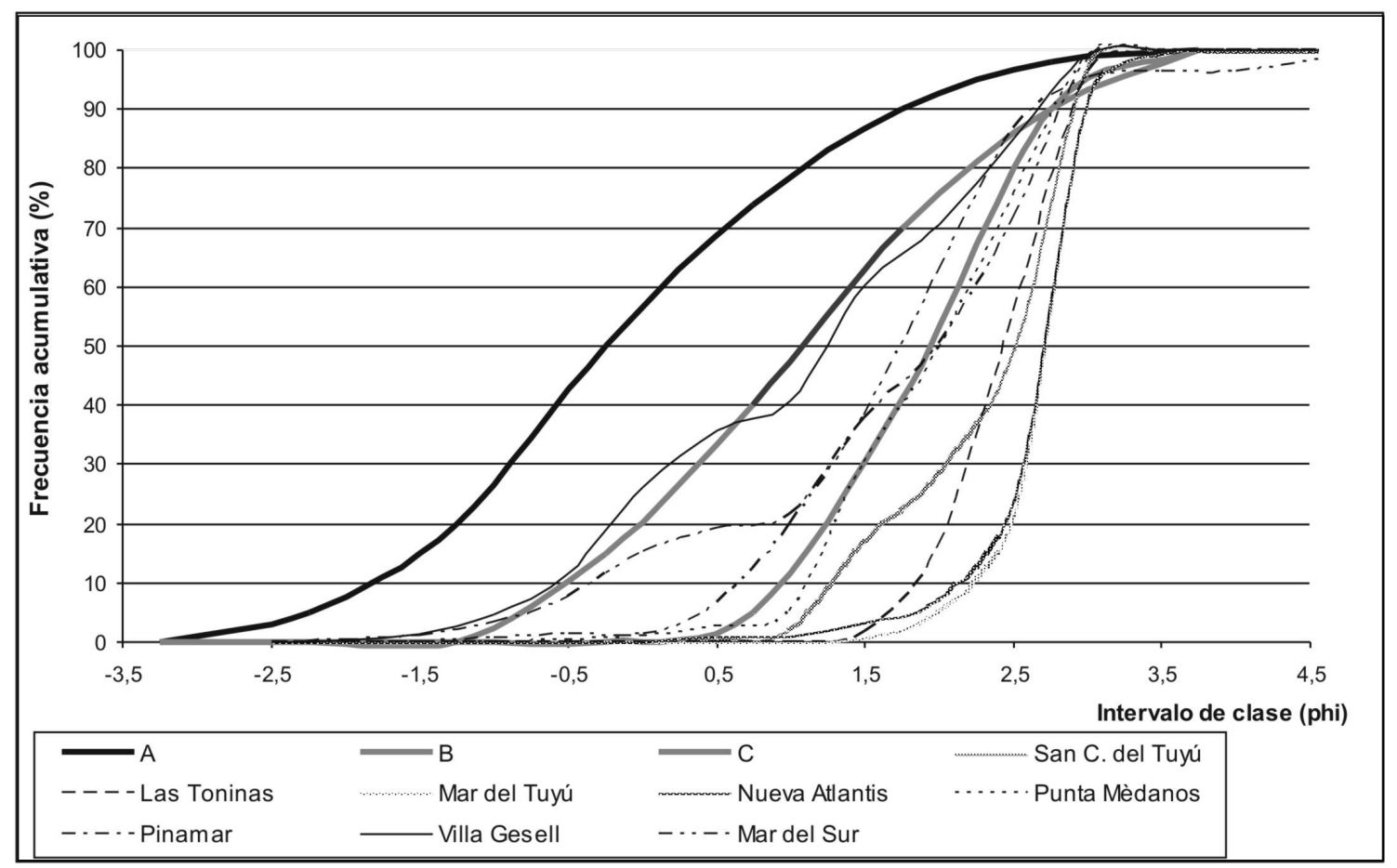

Figura 6 - Límites establecidos para las categorías aptas (A y B) y en obras de "tipo corriente", donde el control de calidad de hormigones se realice en obra (norma IRAM 1627). Los sedimentos tienen la granulometría apta para hormigón cuando sus curvas granulométricas caen dentro de las curvas A y B. En construcciones de poca envergadura se pueden aceptar los sedimentos cuyas curvas esten comprendidas entre A y C.

Los sedimentos correspondientes a las playas de Pinamar tienen un $45 \%$ de los sedimentos con tamaños entre las curvas B y C.

Los sedimentos correspondientes a las playas de San Clemente del Tuyú, Mar del Tuyú, Las Toninas y Punta
Médanos, presentan distribuciones por debajo de la línea $\mathrm{C}$, o sea que son demasiado finas para ser aptas para hormigón.

En todos los casos los sedimentos deberán ser mezclados con fracciones más gruesas para poder tener el tamaño de grano adecuado como para ser empleados como agregado fino para hormigón. 
De lo anteriormente expuesto se concluye que las arenas de playa de la costa de Buenos Aires entre Cabo San Antonio a Necochea no cumplen con las normas IRAM para desarrollo de hormigón, no obstante ello han sido empleadas durante décadas para la construcción edilicia e infraestructura de los municipios costeros. Los importantes retrocesos de la línea de costa y la falta de saturación de la corriente de deriva actual, en especial en áreas urbanizadas, hace imprescindible acabar con las explotaciones clandestinas de arena de playa y duna costera, no solo porque las arenas no son aptas para hormigón y podrían traer problemas estructurales a futuro, sino que también porque intensifican la acción erosiva en todas las localidades aledañas, disminuyendo las tasas de transporte marino y eólico, principales fuentes de abastecimiento del sistema litoral.

\section{Dinamica Costera Y Explotaciones Mineras}

Las labores mineras ejecutadas en el litoral bonaerense han sido desarrolladas sin considerar las condiciones hidrodinámicas y morfológicas de cada sector costero.

Los análisis de las distintas explotaciones mineras a lo largo de la costa han provocado impactos dependiendo de la morfología e hidrodinámica del sistema costero. En todos los casos se evidenció una falta de saturación en la corriente de deriva litoral, lo que hizo que la erosión se traslade como una onda en el sentido de la deriva litoral, es decir hacia el norte para estas costas. El tipo o morfología costera que encuentre esta onda va a ser afectada en mayor o menor grado la erosión a la que este sujeta.

En las costas de dunas las explotaciones han ocasionado un marcado deterioro de la playa, más que un retroceso de la costa. Se ha observado una degradación de la berma, que es la defensa natural frente a las tormentas. El tamaño de grano de los sedimentos que componen la berma es más grueso, razón por la cual es más propicia para la explotación. La ruptura de la berma, sumado a la falta de saturación en sedimentos de las corrientes litorales durante los eventos de tormenta, han convertido a amplios sectores litorales en zonas muy vulnerables a la erosión.

En el caso de costas del tipo A, donde la playa natural no posee bermas, la explotación de arena de playa ha producido importantes retrocesos locales de la línea de costa. Ejemplos de este tipo se pueden observar en Mar de Ajó, Las Toninas norte, San Clemente Sur.

En las costas tipo B, la explotación modificó las dimensiones de la berma y disminuyó el nivel de playa. La atenuación del efecto erosivo producido por las explotaciones en este tipo de costas lo regula la duna costera. Durante las tormentas la arena es tomada del pie de duna para alcanzar la saturación. Es por ello que los mayores efectos erosivos se concentran en aquellas urbanizaciones en cuyos frentes costeros se ha perdido la duna por la urbanización.

En el caso de costas acantiladas cohesivas, la subsaturación de las corrientes ha incrementado las velocidades de retroceso de los acantilados, intensificando el retroceso hacia el sitio de explotación. Las velocidades de retroceso de la costa se incrementaron en un 50 a $80 \%$ para los sitios próximos a las explotaciones (menos de $5 \mathrm{~km}$ ), entre un 20 y un $50 \%$ (hasta $30 \mathrm{~km}$ ), un $5 \%$ a más de $50 \mathrm{~km}$ (Centinela del Mar).

El material arenoso depositado sobre las plataformas de abrasión, que constituye depósitos de playa, es transportado por las corrientes litorales hasta incrementar su saturación en sedimentos, produciendo la degradación de las playas. Las consecuencias producidas por la explotación en este tipo de costa se evidenciaron mediante:

- El hallazgo de plataformas de abrasión desprovistas en su totalidad de los sedimentos arenosos, en sectores que antiguamente conformaban depósitos de playa (Figura 4F).

- Formación de acantilados en dunas actuales.

- Remanentes de dunas aislados en sectores de playa.

- Localización de casas en la zona intermareal (Figura 4F).

- Cortes de senderos vecinales por rápido retroceso de la línea de costa

La intensidad de la erosión, depende también, del efecto de la subsaturación sobre otros factores que regulan el retroceso de los acantilados.

Las condiciones de subsaturación en sedimentos originadas durante y luego de las explotacines mineras han aumentado la acción erosiva del oleaje en las costas cohesivas, y en consecuencia la abrasión, el efecto del martilleo (quarrying) y el efecto hidráulico sobre las fracturas.

El incremento en el efecto abrasivo, se debe a la disminución del espesor de arena que recubren las plataformas. Ya que aumentará el efecto de martilleo de los clastos sobre la misma. La acción del oleaje directamente sobre las diaclasas se incrementa a medida que aumenten la superficie de las plataformas descubiertas de sedimentos.

La subsaturación en sedimentos, sumado al incremento del tamaño de grano sobre las plataformas de abrasión podría afectar la presencia de organismos variando las tasas de bioerosión.

La falta de la cobertura de arena y la repetida exposición de la plataforma rocosa y acantilado a las condiciones subaéreas y subácuas por efecto de las mareas incrementará la meteorizacón física y química.

Asimismo, por la pérdida del espesor de playa que recubre la plataforma la exposición de la base del acantilado al oleaje se incrementará. 


\section{Recuperación}

En costas cohesivas, la suspensión de la actividad minera modificó rápidamente las tasas de retroceso de la línea de costa disminuyendo, su valor, en los períodos posteriores al cese de la explotación. Los sectores urbanizados presentaron recuperaciones menos efectivas.

Las tasas de retroceso se elevaron por la explotación en un $88 \%$ en el área de extracción de la arenera en el Partido de Lobería. Las tasas de retroceso en San Jacinto (Partido de General Pueyrredón), se redujeron en un $44 \%$ entre 1985 1988 y a un 66\% 1988-1992 por el cese de la explotación. La suspención de la explotación sumado a una notable reducción en la actividad de la industria para la construcción ocasionaron una disminución en las tasas de retroceso, las cuales variaron de 5 m/año en 1970-1982, a 3,33 m/año en 1985- 1988 y 2,20 m/año de 1988 a 1992 (Cionchi et al., 1993).

En líneas generales se pudo observar que las velocidades de retroceso de la costa se aceleran o retardan en igual medida durante la explotación y recuperación respectivamente; es decir que la recuperación por interrupción de las actividades mineras es semejante en magnitud a la erosión inducida durante la explotación.

Asimismo, en costas de dunas se ha observado una rápida recuperación de las bermas estables y transicionales, $\mathrm{y}$ un incremento del nivel de playa luego del cese de la explotación (San Clemente, Mar de Ajó, Villa Gesell).

La intensa erosión que está afectando gran parte de las localidades costeras de la provincia de Buenos Aires, demuestra que las playas están en un estado crítico. Las acciones humanas sobre el sistema han intensificado la erosión en los centros urbanos y la explotación de arena ha sido uno de los principales detonantes. Es por ello que es necesario interrumpir definitivamente la explotación de arena de playa y duna costera, ya que a pesar de las prohibiciones se sigue realizando un manejo inadecuado en distintos municipios.

Las ondas erosivas generadas por la explotación se desplazan varios kilómetros desde el foco de explotación. La longitud de playa afectada depende del tipo de costa y del grado de abastecimiento del sistema litoral, y se han detectado desplazamientos de hasta $50 \mathrm{~km}$ en playas correspondientes a costas cohesivas. Es por ello imprescindible parar la explotación, no solo de las áreas urbanizadas, sino que también, de aquellas en estado natural, ya que las mismas constituyen el reservorio natural de ingreso de arena al sistema litoral.

Los requerimientos de arena son múltiples. A nivel de dependencias de Obras Públicas municipales se la emplea para el mantenimiento de calles, de relleno, no necesitan de áridos calificados y por lo tanto podrían emplearse otras fuentes de aporte. Por ejemplo el Municipio de La Costa reutiliza aproximadamente $30.000 \mathrm{~m}^{3}$ al año, de arena que invade sus avenidas costaneras e infraestructura edilicia.

Los cordones litorales y los campos de dunas continentales presentan características mineralógicas similares a los sectores de playa y duna costera respectivamente, pero su granulometría es difenrente, en especial si se los compara con los sedimentos de playa actual (berma). Estos cambios granulométricos podrían compensarse efectuando mezclas in situ con sedimentos correspondientes a intervalos más gruesos (niveles de conchilla) o simplemente asignándoles otros usos (rellenos de zonas bajas, reboque, etc), que en la actualidad se emplea la arena de playa.

\section{Conclusiones}

Las explotaciones mineras de arena de playa han afectado de manera diferente a las localidades balnearias, dependiendo fundamentalmente de la morfología e hidrodinámica que caracteriza cada tipo de costa. En las playas localizadas entre Punta Rasa y Punta Médano, donde la playa natural no posee bermas muy marcadas, la explotación de arena ha producido importantes retrocesos locales en la línea de costa. Ejemplos de este tipo se pueden observar en Mar de Ajó, Las Toninas norte y San Clemente Sur. En la costa entre Punta Médano y Mar Chiquita (B), la explotación modificó las dimensiones de la berma y disminuyó el nivel de playa. Las costas acantiladas cohesivas fueron las más impactadas, ya que el abastecimiento de arena es más restringido. Se detectó un importante incremento en las tasas de retroceso de los acantilados, pérdida de playa y modificación de los procesos locales que intervienen directamente en relación al poder del oleaje. Se incrementó asimismo la erosión por aumento de la meteorización física del oleaje, medianete el aumento del efecto martillo (quarrying) sobre acantilados y plataformas de abrasión y el efecto hidráulico del oleaje sobre las grietas. Las explotaciones mineras autorizadas llegaron a explotar volúmnes de arena de $400.000 \mathrm{a} 600.000 \mathrm{~m}^{3}$ anuales.

Las características granulométricas de las arenas de playa demostraron que ninguna localidad presenta arenas en con tamaño de grano apropiado para ser empleado para hormigón. No obstante ello, las arenas de playa de Villa Gesell y Mar del Sur son las que más se ajustan a las normas IRAM. El resto de las playas no cumple con los intevalos mínimos.

El alto impacto ambiental que ha generado la actividad minera en la zona costera, sumado a la inapropiada calidad del árido (arena de playa) para la construcción, hacen necesaria la búsqueda de sitios o metodologías de explotación alternativas en el Litoral Atlántico Bonaerense. Asimismo la baja incidencia registrada en las economías locales por el cese de las explotaciones, son evidencias que indican que la extracción de arena de playa y duna costera no deberían ser una opción aceptable si se pone en riesgo el principal recurso 
económico de estas localidades que es sin lugar a dudas la playa.

Es necesario contar con nuevas fuentes de abastecimiento de arena que podrían provenir del sector continental (antiguas líneas de costa o dunas inactivas) o de la playa sumergida.

\section{Agradecimientos}

Los autores desean expresar su más sincero agradecimiento a los árbitros de este trabajo cuyas sugerencias y correcciones han sido de gran importancia para mejorar la calidad del mismo. Las investigaciones fueron financiadas por el subsidio otorgado por la Universidad de Buenos Aires, UBACyT X083.

\section{Referencias Bibliograficas}

Anctil, F. \& Ouellet, Y., (1990). Preliminary evaluation of impacts of sand extraction near Iles-de-la Madeleine Archipielago, Quebec, Canada. Journal of Coastal Research, 6, 37-51.

Caballé, M. F., Ganuza, D, Corriale, N, Almonacid, M.B., (2005). Recursos arenosos en el delta del Paraná y Litoral Atlántico. Relatorio del XVI Congreso Geológico Argentino, Eds: Barrio R.E., Etcheverry, R.O., Caballé, M.F., Llambias, E.La Plata, 435-440.

Cionchi, J.L.; Alvarez, J.R.; Del Río, J.L.; Ferrante, A., (1993). El efecto antrópico en el retroceso de la linea de costa del Partido de General Pueyrredón (provincia de Buenos Aires). Actas Del $12^{\circ}$ Congreso Geológico Argentino Y $2^{\circ}$ Congreso De Explotación De Hidrocarburos. 6: 318-322. Buenos Aires.

Hilton, M.J., (1989). Management of the New Zealand coastal sand mining industry: Some implications of a geomorphic study of the Pakiri sand body. New Zealand Geographer, 45, 14-25.

Hilton, M.J., (1990). Applying the principle of sustainability to coastal sand mining:The case of PakiriMangawhai Beach, New Zealand. Environmental Management., 18, 17-24.

Hesp, P. \& Hilton, M. J. (1996). Nearshore-surfzone system limits and the impact of sand extraction. Journal of Coastal Research, 12(3):726-747.

Iram , (1997). Norma 1627, Agregados, granulometría de los agregados para hormigones. Instituto Argentino de Normalización, 1-23.
Isla, F.I. \& Fasano, J.L., (1987). Arenas litorales de la provincia de Buenos Aires, recurso minero o recurso ambiental. X Congreso Geológico Argentino, Actas, I: 79-82.

Isla, F., (1997). Seasonal behaviour of Mar Chiquita tidal inlet in relation to adjacent beaches, Argentina. Journal of Coastal Research 13, 4, 1221-1232.

Lopez, R.A. \& Marcomini, S.C., 2003. Extraer onoarena: ¿Es esa una cuestión? V Jornadas Nacionales De Ciencias Del Mar. Mar del Plata, Resúmenes, 132 p.

Marcomini, S.C. y Lopez, R.A. (1997). Influencia de la urbanización en la dinámica costera, Villa Gesell, provincia de Buenos Aires, República Argentina. Revista de la Asociación Argentina de Sedimentología., 4 (2):79-96.

Marcomini, S.C. \& López, R.A., (1999)Alteración de la dinámica costanera por efecto de la explotación de arena de playa, partido de General Alvarado, provincia de Buenos Aires. Revista de la Asociación Argentina de Sedimentología.

Schnack, E.J.; Alvarez, J.R.., Cionchi, J.L. (1983). El carácter erosivo de la línea de costa entre Mar Chiquita y Miramar, provincia de Buenos Aires. En: Simpósio: "Oscilaciones Del Nivel Del Mar Durante El Último Hemiciclo Deglacial En La Argentina", Actas: 118130. Mar del Plata.

Tsurusaki, K.; Takashi, I; Arita, M. (1988). Seabed sand mining in Japan. Marine Mining, 7, 49-67.

Uda, T.; Agemori, C.; Chujo, N. (1986). Beach changes caused by offshore dredging. Coastal Engineering in Japan, 29, 215-226.

Williams, S.J. \& Cichon, H.A. (1993). Geologic assessments and characterization of marine sand resources Gulf of Mexico Region. En: Coastalines of the Gulf of Mexico (Eds): S, Lasca y A. Puffer. 217-223. 